\title{
A methanol line survey toward high-mass star-forming regions ${ }^{\star}$
}

\author{
V. Minier ${ }^{1,2}$ and R. S. Booth ${ }^{2}$ \\ 1 Department of Astrophysics and Optics, School of Physics, University of New South Wales, NSW 2052, Australia \\ 2 Onsala Space Observatory, 43992 Onsala, Sweden
}

Received 7 January 2002 / Accepted 14 February 2002

\begin{abstract}
We present the results of a search for methanol maser and thermal lines in 11 transitions in the range $85-112 \mathrm{GHz}$ toward 23 star-forming regions exhibiting class I and class II methanol masers. The selected frequencies are 85.5, 86.6, 94.5, 95.1, 96.7 (quartet line series), 107.0, 108.8 and 111.2 GHz. Five masers were confirmed at $107.0 \mathrm{GHz}$ while new masers were found at 85.5, 86.6 and 108.8 GHz. Many detected emission lines have a quasi-thermal origin. The detection rates of methanol emission are high at $95.1 \mathrm{GHz}(87 \%)$ and $96.7 \mathrm{GHz}$ $(96 \%)$, satisfactory at 107.0 and $108.9 \mathrm{GHz}(\sim 50 \%)$ while the detection rates at 85.5, 94.5 and $111.3 \mathrm{GHz}$ are low $(\sim 20 \%)$. Most reported $95.1 \mathrm{GHz}$ emission is masing.
\end{abstract}

Key words. masers - stars: formation - stars: circumstellar matter

\section{Introduction}

Methanol masers are classified in two categories based on their location in star-forming regions (Menten 1991a) and their pumping mechanism (Sobolev 1993). Class I methanol masers are generally observed far away $\left(\sim 10^{4} \mathrm{AU}\right)$ from the young massive stars and they are believed to arise at the shocked interface between the outflows and the interstellar medium. Class I methanol masers have been detected at 25.0, 36.1, 44.0, 84.5, 95.1 and $146.6 \mathrm{GHz}$ (e.g. Turner et al. 1972; Haschick et al. 1990; Plambeck \& Wright 1988). More recently, Kalenskii et al. (2001) discovered using the Onsala-20 m telescope two class I methanol masers at 84.5 and $95.1 \mathrm{GHz}$ in the low-mass star region L-1157 mm. It might be the first detection of a methanol maser associated with an outflow from a low-mass star. In contrast, class II methanol masers lie in the near vicinity (within $2000 \mathrm{AU}$ ) of young massive stars. For instance, the two brightest class II methanol masers at 6.7 and $12.2 \mathrm{GHz}$ (Menten 1991b; Batrla et al. 1987 ) are directly associated with very early signposts of massive star formation such as the hot molecular cores, the inner ionised part of jets and the hyper compact $\mathrm{H}$ II regions (Minier et al. 2001).

Following the discovery of 6.7 and $12.2 \mathrm{GHz}$ methanol masers, Sobolev et al. (1997) have proposed a model that predicts several other maser lines in the range 6-250 GHz. Many of these predicted transitions are effectively masing

\section{Send offprint requests to: V. Minier,}

e-mail: vminier@bat.phys.unsw.edu.au

* Table A1 and Figs. A1-A2 of Appendix are only available in electronic form at http://www.edpsciences.org and were detected in the past ten years at 23.1, 37.7, 38.2, 38.4, 85.5, 86.6, 86.9, 107.0, 108.8, 156.6, 157.2 GHz (e.g. Menten \& Batrla 1989; Haschick et al. 1989; Val'tts et al. 1995; Caswell et al. 2000) and maser lines are predicted at for example 94.5 and $111.2 \mathrm{GHz}$. New maser lines at $85.5,86.6,86.9$ and $108.8 \mathrm{GHz}$ have been detected very recently by Cragg et al. (2001) in G9.62+0.20, by Sutton et al. (2001) in W3(OH), and by Val'tts et al. (1999) in G345.01+1.79, respectively.

In the present paper, the results of a large search for new class II maser lines toward 23 sources are given. These sources are believed to harbour young massive stars at the earliest stage of their lives. The frequency selection is based on Sobolev et al.'s predictions of intense maser lines and the selected frequencies for this search are listed in Table 1. The secondary purpose of this work is to derive the rotational temperature and the column density of the methanol clouds using the $96.7 \mathrm{GHz}$ quartet line series as a probe. Finally, a class I methanol maser at $95.1 \mathrm{GHz}$ has also been searched in order to further investigate the connection between the two distinct classes of masers. All the observed transitions are listed in Table 1.

\section{Observations and summary of the results}

The observations were carried out in November 1997, February 1998, May 1998 and November 1998 using the Onsala- $20 \mathrm{~m}$ telescope. Only transitions in the frequency range $85-112 \mathrm{GHz}$ were observed. About one hour per transition was spent for each source, including calibration and integration time on source in a dualbeam switching mode. For these observations, both the 
Table 1. Observed methanol line transitions.

\begin{tabular}{llll}
\hline \hline Transition & $\begin{array}{l}\text { Frequency } \\
(\mathrm{MHz})\end{array}$ & $\begin{array}{l}E u / k \\
(\mathrm{~K})\end{array}$ & $\begin{array}{l}\text { Maser }(\mathrm{M}) \text { or } \\
\text { Thermal }(\mathrm{T})\end{array}$ \\
\hline $6_{-2} \rightarrow 7_{-1} \mathrm{E}$ & 85568.084 & 74.7 & $\mathrm{M}$ \\
$7_{2} \rightarrow 6_{3} \mathrm{~A}^{-}$ & 86615.602 & 102.7 & $\mathrm{M}$ \\
$8_{3} \rightarrow 9_{2} \mathrm{E}$ & 94541.778 & 131.3 & $\mathrm{M}$ \\
$8_{0} \rightarrow 7_{1} \mathrm{~A}^{+}$ & 95169.440 & 83.6 & $\mathrm{M}$ \\
$2_{-1} \rightarrow 1_{-1} \mathrm{E}$ & 96739.39 & 12.6 & $\mathrm{~T}$ \\
$2_{0} \rightarrow 1_{0} \mathrm{~A}^{+}$ & 96741.42 & 7.0 & $\mathrm{~T}$ \\
$2_{0} \rightarrow 1_{0} \mathrm{E}$ & 96744.58 & 20.1 & $\mathrm{~T}$ \\
$2_{1} \rightarrow 1_{1} \mathrm{E}$ & 96755.51 & 28.0 & $\mathrm{~T}$ \\
$3_{1} \rightarrow 4_{0} \mathrm{~A}^{+}$ & 107013.812 & 28.3 & $\mathrm{M}$ \\
$0_{0} \rightarrow 1_{-1} \mathrm{E}$ & 108893.948 & 13.1 & $\mathrm{M}$ \\
$7_{2} \rightarrow 8_{1} \mathrm{~A}^{+}$ & 111289.515 & 102.7 & $\mathrm{M}$ \\
\hline
\end{tabular}

filter bank (64 MHz bandwidth and velocity resolution of $\sim 0.75 \mathrm{kms}^{-1}$ ) and the autocorrelator (40 and $80 \mathrm{MHz}$ bandwidths and velocity resolutions of 0.07 and $0.15 \mathrm{~km} \mathrm{~s}^{-1}$, respectively) were used. Due to different observing epochs and thus weather conditions, the rms of the noise level varies from 10 to $200 \mathrm{mK}$ and is generally lower than $80 \mathrm{mK}$. Complementary observations of $\mathrm{C}^{18} \mathrm{O}(1-0)$ and $\mathrm{NH}_{3}(1,1)$ were also carried out toward a limited number of sources. The goal of these observations was to compare the line-of-sight velocity of the methanol spectral features with those of other species. The gain of the Onsala-20 $\mathrm{m}$ telescope is $\sim 20 \mathrm{Jy} \mathrm{K}^{-1}$ in the range 85-112 GHz.

The source sample consists of 23 sources taken from Menten (1991b) and Val'tts et al. (1995). Fifteen of these sources have been imaged at 6.7 and $12.2 \mathrm{GHz}$ using VLBI techniques (Minier et al. 2000, 2001). Many target sources are class II methanol maser sites, while some of them exhibit class I methanol masers. Orion $\mathrm{KL}$ is for example the prototypical class I methanol maser source while $\mathrm{W} 3(\mathrm{OH})$ is the richest object in class II maser transitions. All these sources are believed to be closely connected to high-mass star-forming regions. The source coordinates are listed in Table 2. A summary of the detection is given in Table 2 as well. The derived Gaussian parameters and spectra of the detected lines are presented in Appendix A. Complementary information on the other molecular lines detected toward the selected methanol sources is also given in Table A.1.

Three groups of sources are seen (Table 2). The first group is composed of sources exhibiting class II masers at one of the predicted frequencies. They also emit 6.7 GHz masers. Nine of them are typical class II methanol maser sources: W3 $(\mathrm{OH})$, AFGL 5180 (also known as S252 and G188.95+0.89), G29.95-0.02, W48, W51-IRS1, G59.78+0.06, W75N, CepA and NGC 7538-IRS1. The tenth one, DR21(OH), is known as a class I methanol maser source. The second group consists of six sites exhibiting only thermal emission in one predicted maser line transition, i.e. broad features of $F W H M \sim 2$ to $11 \mathrm{kms}^{-1}$. Orion KL and W51e2 are typical representa- tives of this group. The third group contains seven sources without any emission detected at the predicted class II maser frequencies. Many of these sources, however, exhibit thermal emission at $96.7 \mathrm{GHz}$. S255, MonR2 and IRAS $20126+4104$ are in this group. Finally, many sources exhibit thermal emission and/or masers at 95.1 GHz.

The most detected transitions (see Table 2) are at 95.1 GHz (87\%), 96.7 GHz (96\%), $107 \mathrm{GHz}(48 \%)$ and $108.8 \mathrm{GHz}(43 \%)$. Class II masers are poorly detected at all frequencies except perhaps at $107.0 \mathrm{GHz}$ with 6 cases. In contrast, strong thermal methanol emission is well present at $96.7,107.0$ and $108.8 \mathrm{GHz}$; these lines correspond to low excitation energies with an upper energy state $\left(E_{\mathrm{u}} / k\right)$ lower than $30 \mathrm{~K}$. Thermal emission at 85.5 , 94.5 and $111.2 \mathrm{GHz}$ corresponding to higher excitation lines $\left(74<E_{\mathrm{u}} / k<132 \mathrm{~K}\right)$ is poorly detected. Finally, 95.1 GHz masers are massively observed in both class I and class II methanol maser sources.

\section{Detection of new class II masers}

In this section, a more detailed analysis of the class II maser line candidates is presented. In order to be identified as maser features, detected spectral lines have to fulfil conditions related to their line width, line-of-sight velocity and intensity.

First an unsaturated maser feature is expected to have a line width smaller than the thermal line width, i.e. smaller than $0.4 \mathrm{~km} \mathrm{~s}^{-1}$ for methanol. However, a spectral feature of $F W H M$ greater than $0.4 \mathrm{~km} \mathrm{~s}^{-1}$ could also have a maser origin. A broad spectral line could, for example, be a blend of unresolved and narrow maser features. Such a spectral profile has been seen in the 6.7 and $12.2 \mathrm{GHz}$ methanol masers where some maser features have a $F W H M$ of $\sim 2 \mathrm{~km} \mathrm{~s}^{-1}$ consisting of many partially resolved and narrower features. An example of such a spectral morphology is the $12.2 \mathrm{GHz}$ methanol spectrum in NGC 7538 (Minier et al. 2002, hereafter MBC02).

Secondly, by comparing for a given source the peak velocity and line width of a detected line with those of the $6.7 \mathrm{GHz}$ maser spectral features and thermal emission lines, it is possible to further differentiate between maser candidates and possible thermal emission. For instance, a maser feature is expected to be narrower than a quasithermal line.

Finally, a methanol maser is expected to be a bright radiation that originates from very small structures (e.g. $<50$ mas for $12.2 \mathrm{GHz}$ masers, MBC02) seen as point sources in the large beam of a single dish telescope $\left(\theta_{\text {beam }} \simeq 30\right.$ arcsec $)$. Taking the predicted brightness temperatures $\left(T_{\mathrm{b}}>10^{5} \mathrm{~K}\right)$ in Sobolev et al. (1997) and assuming that all the class II methanol masers arise from structures as small as those producing the $12.2 \mathrm{GHz}$ masers, the maser candidates could produce antenna temperatures in the range 0.01 to $10 \mathrm{~K}$. Most of the masers should be detectable with the sensitivity of these observations (see rms noise level in Table A.1). 
Table 2. Source list: observed positions, frequencies and summary of the detection; $y, n$ and NO mean detected, not detected and not observed, respectively; M means maser. Note: ${ }^{1}$ The maser source in AFGL 5180 is often named G188.95+0.89 and S252 in the literature (e.g. Menten 1991b); ${ }^{2}$ observed by others (see Table A.1).

\begin{tabular}{|c|c|c|c|c|c|c|c|c|c|c|}
\hline \multirow[t]{2}{*}{ Sources } & \multicolumn{2}{|c|}{ Coordinates (J2000) } & \multicolumn{8}{|c|}{ Frequency $(\mathrm{GHz})$} \\
\hline & $\overline{\mathrm{RA}(\mathrm{h} \mathrm{m} \mathrm{s})}$ & 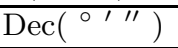 & 85.5 & 86.6 & 94.5 & 95.1 & 96.7 & 107.0 & 108.8 & 111.2 \\
\hline $\mathrm{W} 3(\mathrm{OH})$ & 022704.7 & 615225 & $\mathrm{y}$ & $\mathrm{y}(\mathrm{M})$ & $\mathrm{n}$ & $\mathrm{y}$ & $\mathrm{y}$ & $\mathrm{y}(\mathrm{M})$ & $\mathrm{y}$ & $\mathrm{y}$ \\
\hline Orion S6 & 053512.2 & -052406 & $\mathrm{n}$ & $\mathrm{n}$ & $\mathrm{n}$ & $\mathrm{y}$ & $\mathrm{y}$ & $\mathrm{y}$ & $\mathrm{n}$ & $\mathrm{n}$ \\
\hline Orion KL & 053514.5 & -052229 & $\mathrm{y}$ & $\mathrm{y}$ & $\mathrm{y}$ & $\mathrm{y}(\mathrm{M})$ & $\mathrm{y}$ & $\mathrm{y}$ & $\mathrm{y}$ & $\mathrm{y}$ \\
\hline OMC2 & 053527.5 & -050936 & $\mathrm{n}$ & $\mathrm{n}$ & $\mathrm{n}$ & $\mathrm{y}(\mathrm{M})$ & $\mathrm{y}$ & $\mathrm{n}$ & $\mathrm{n}$ & $\mathrm{n}$ \\
\hline $\mathrm{S} 231$ & 053912.9 & 354554 & $\mathrm{n}$ & $\mathrm{n}$ & $\mathrm{n}$ & $\mathrm{y}(\mathrm{M})$ & $\mathrm{y}$ & $\mathrm{n}$ & $\mathrm{y}$ & $\mathrm{n}$ \\
\hline MonR2 & 060746.3 & -062309 & $\mathrm{n}$ & $\mathrm{n}$ & $\mathrm{n}$ & $\mathrm{n}$ & $\mathrm{y}$ & $\mathrm{n}$ & $\mathrm{n}$ & $\mathrm{n}$ \\
\hline AFGL $5180^{1}$ & 060854.2 & 213837 & $\mathrm{n}$ & $\mathrm{n}$ & $\mathrm{n}$ & $\mathrm{y}$ & $\mathrm{y}$ & $\mathrm{y}(\mathrm{M})$ & $\mathrm{n}$ & $\mathrm{n}$ \\
\hline $\mathrm{S} 255$ & 061256.4 & 175954 & $\mathrm{n}$ & $\mathrm{n}$ & $\mathrm{n}$ & $\mathrm{y}(\mathrm{M})$ & $\mathrm{y}$ & $\mathrm{n}$ & $\mathrm{n}$ & $\mathrm{n}$ \\
\hline S269 & 061436.5 & 134941 & $\mathrm{n}$ & $\mathrm{n}$ & $\mathrm{n}$ & $\mathrm{n}$ & $\mathrm{n}$ & $\mathrm{n}$ & $\mathrm{n}$ & $\mathrm{n}$ \\
\hline G29.95-0.02 & 184603.9 & -023921 & $\mathrm{y}(\mathrm{M})$ & $\mathrm{n}$ & $\mathrm{n}$ & $\mathrm{NO}\left(\mathrm{M}^{2}\right)$ & $\mathrm{y}$ & $\mathrm{n}$ & $\mathrm{n}$ & $\mathrm{n}$ \\
\hline W48 & 190146.2 & 011341 & $\mathrm{n}$ & $\mathrm{n}$ & $\mathrm{y}$ & $\mathrm{NO}$ & $\mathrm{y}$ & $\mathrm{y}(\mathrm{M})$ & $\mathrm{n}$ & $\mathrm{n}$ \\
\hline W51-IRS1 & 192342.1 & 143041 & $\mathrm{y}$ & $\mathrm{y}(\mathrm{M})$ & $\mathrm{y}$ & $\mathrm{y}$ & $\mathrm{y}$ & $\mathrm{y}$ & $\mathrm{y}$ & $\mathrm{n}$ \\
\hline W51e2 & 192343.9 & 143036 & $\mathrm{y}$ & $\mathrm{y}$ & $\mathrm{y}$ & $\mathrm{y}(\mathrm{M})$ & $\mathrm{y}$ & $\mathrm{y}$ & $\mathrm{y}$ & $\mathrm{y}$ \\
\hline W51met1 & 192343.9 & 142925 & $\mathrm{n}$ & $\mathrm{n}$ & $\mathrm{n}$ & $\mathrm{y}(\mathrm{M})$ & $\mathrm{y}$ & $\mathrm{n}$ & $\mathrm{n}$ & $\mathrm{n}$ \\
\hline G59.78+0.06 & 194310.9 & 234403 & $\mathrm{n}$ & $\mathrm{n}$ & $\mathrm{n}$ & $\mathrm{y}(\mathrm{M})$ & $\mathrm{y}$ & $\mathrm{n}$ & $\mathrm{y}(\mathrm{M})$ & $\mathrm{n}$ \\
\hline ON1 & 201009.2 & 313135 & $\mathrm{n}$ & $\mathrm{n}$ & $\mathrm{n}$ & $\mathrm{y}(\mathrm{M})$ & $\mathrm{y}$ & $\mathrm{n}$ & $\mathrm{y}$ & $\mathrm{n}$ \\
\hline IRAS $20126+4104$ & 201426.0 & 411340 & $\mathrm{n}$ & $\mathrm{n}$ & $\mathrm{n}$ & $\mathrm{y}(\mathrm{M})$ & $\mathrm{y}$ & $\mathrm{n}$ & $\mathrm{n}$ & $\mathrm{n}$ \\
\hline ON2 & 202142.6 & 372608 & $\mathrm{n}$ & $\mathrm{n}$ & $\mathrm{n}$ & $\mathrm{y}(\mathrm{M})$ & $\mathrm{y}$ & $\mathrm{n}$ & $\mathrm{n}$ & $\mathrm{n}$ \\
\hline W75N & 203836.8 & 423759 & $\mathrm{n}$ & $\mathrm{n}$ & $\mathrm{n}$ & $\mathrm{y}(\mathrm{M})$ & $\mathrm{y}$ & $\mathrm{NO}\left(\mathrm{M}^{1}\right)$ & $\mathrm{n}$ & $\mathrm{n}$ \\
\hline DR21(OH) & 223900.7 & 422251 & $\mathrm{y}(\mathrm{M})$ & $\mathrm{n}$ & $\mathrm{n}$ & $\mathrm{y}(\mathrm{M})$ & $\mathrm{y}$ & $\mathrm{y}$ & $\mathrm{y}$ & $\mathrm{n}$ \\
\hline CepA & 225618.1 & 620149 & $\mathrm{n}$ & $\mathrm{n}$ & $\mathrm{n}$ & $\mathrm{y}(\mathrm{M})$ & $\mathrm{y}$ & $\mathrm{y}(\mathrm{M})$ & $\mathrm{n}$ & $\mathrm{n}$ \\
\hline NGC 7538-IRS1 & 231345.4 & 612810 & $\mathrm{n}$ & $\mathrm{y}$ & $\mathrm{n}$ & $\mathrm{y}(\mathrm{M})$ & $\mathrm{y}$ & $\mathrm{y}(\mathrm{M})$ & $\mathrm{y}$ & $\mathrm{n}$ \\
\hline NGC $7538-44 \mathrm{GHz}$ & 231346.4 & 612733 & $\mathrm{n}$ & $\mathrm{n}$ & $\mathrm{n}$ & $\mathrm{y}(\mathrm{M})$ & $\mathrm{y}$ & $\mathrm{y}$ & $\mathrm{y}$ & $\mathrm{n}$ \\
\hline Total & detection & rate $(\%)$ & 26 & 22 & 17 & 87 & 96 & 48 & 43 & 13 \\
\hline Maser & detection & rate $(\%)$ & 9 & 9 & 0 & 70 & 0 & 26 & 4 & 0 \\
\hline
\end{tabular}

Using the above criteria and Table A.1 for selecting maser lines, new possible masers are reported at 85.5, 86.6 and $108.8 \mathrm{GHz}$ while five maser detections at $107.0 \mathrm{GHz}$ in Val'tts et al. (1995) and one 86.6 GHz maser in W3(OH) (Sutton et al. 2001) are confirmed. All the detected maser lines agree in velocity with their $6.7 \mathrm{GHz}$ maser counterparts (Table A.1). The maser spikes have FWHM of 0.11 to $1.88 \mathrm{~km} \mathrm{~s}^{-1}$. The new maser detections are two 85.5 GHz masers in G29.95-0.02 and in DR21(OH), one 86.6 GHz masers in W51-IRS1, and one $108.8 \mathrm{GHz}$ maser in G59.78+0.06. All these masers have a low intensity from 0.08 to $0.15 \mathrm{~K}$ or 1.6 to $3.0 \mathrm{Jy}$. This corresponds to detections at a level of 3 to $5-\sigma$. They need to be confirmed and monitored to detect any variability. The detection of weak masers and more generally the low detection rate of our survey are puzzling as many maser models predict maser intensities exceeding our sensitivity. Given the large number of targeted sources, it is for instance surprising that only two sources exhibit masers at 85.5 and $86.6 \mathrm{GHz}$. Among the available maser models, new modelling by Cragg et al. (2001) shows that $86.6 \mathrm{GHz}$ masers require low density $\left(\sim 10^{8} \mathrm{~K}\right.$ for $\left.<10^{6} \mathrm{~cm}^{-3}\right)$ while 85.5, 94.5, 108.8 and $111.2 \mathrm{GHz}$ maser intensities reach their maxima $\left(\sim 10^{7}-10^{9} \mathrm{~K}\right)$ for density between $10^{6}$ and $10^{8} \mathrm{~cm}^{-3}$. Our generally poor detection rate would imply very low densities in many sources to confirm the recent methanol maser models.

In four sources (W3(OH), W51-IRS1, G59.78-0.06 and NGC 7538-IRS1), the masers are observed on the top of broader features that could be thermal emission lines (Table A.1). However, Sutton et al. (2001) have interpreted the spectral pedestal components for several methanol lines observed toward $\mathrm{W} 3(\mathrm{OH})$ as produced by a blend of weak masers. The pedestal component would differ from the narrow spike emission through distinct beaming effects and would originate from more spherical clumps. If their interpretation is correct, the broad lines seen at 85.5, 86.6, 107.0, 108.8 and $111.2 \mathrm{GHz}$ in $\mathrm{W} 3(\mathrm{OH})$ are indeed maser lines. Sutton et al. proposed to investigate the nature of the pedestal component with the rotation diagram method. A similar method (Nummelin 1998) is used in this paper to study the pedestal emission for W3(OH), W51-IRS1, G59.78-0.06 and NGC 7538-IRS1. The rotation diagrams are shown in Fig. 1. For W3 $(\mathrm{OH})$, the points corresponding to the emission at 85.5, 96.7, 107.0 and $108.8 \mathrm{GHz}$ fall nicely on a line. They are characterised by a thermal distribution at a temperature of $42 \mathrm{~K}$. This is similar to the rotation temperature $\left(T_{\text {rot }}=45 \mathrm{~K}\right)$ derived by Sutton et al. from the $J_{0} \rightarrow J_{-1}$ series. In contrast, the points corresponding to the emission at 86.6, 
95.1 and 111.2 GHz are off the lines. Based on the rotation diagram, the broad components seen at 85.5, 107.0 and $108.8 \mathrm{GHz}$ could have a quasi-thermal origin while those at 86.6, 95.1 and $111.2 \mathrm{GHz}$ could be due to maser action. Note that additional emission lines at 85.5 and $111.2 \mathrm{GHz}$ are detected around $v_{\mathrm{lsr}}=-51 \mathrm{kms}^{-1}$. In the cases of W51-IRS1 and NGC 7538-IRS1, the spectral pedestal component is seen at 86.6 and $107.0 \mathrm{GHz}$, respectively. From the rotation diagrams, the pedestal components in W51-IRS1 and NGC 7538-IRS1 are likely quasi-thermal lines. The case of G59.78-0.06 is more complex. It is not clear whether the methanol line observed at 108.8 $\mathrm{GHz}$ is purely thermal (108.8 (M+T) in Fig. 1) or whether it consists of a maser spike (108.8 (M) in Fig. 1) on a top of a broader pedestal component (108.8 (T) in Fig. 1).

Finally, assuming that all class II methanol masers originate from the same masing regions in a given site, the angular sizes $\left(\theta_{\mathrm{S}}\right)$ of the masing structures is approximated with those found at $12.2 \mathrm{GHz}$ in MBC02. Taking for $\theta_{\mathrm{S}}$ a mean value of 10 mas, the brightness temperature is derived for each detected maser. The results are given in Table 3. The brightness temperatures range from $10^{6.2}$ to $10^{7.8} \mathrm{~K}$. The highest $T_{\mathrm{b}}$ are found at $107.0 \mathrm{GHz}$. These values agree with those predicted by Sobolev et al. (1997).

To sum up the methanol lines at 85.5, 86.6, 107.0 and $108.8 \mathrm{GHz}$ in W3(OH), AFGL 5180, G29.95-0.02, W48, W51-IRS1, G59.78+0.06, DR 21(OH), CepA and NGC 7538 and listed in Table 3, are likely maser lines.

Table 3. Brightness temperatures of class II masers: $T_{\mathrm{b}}$ is derived from the flux density of the maser peak and assuming angular dimensions of 10 mas for the masing regions as measured at $12.2 \mathrm{GHz}(\mathrm{MBC} 02)$.

\begin{tabular}{lll}
\hline \hline Sources & Frequency $(\mathrm{GHz})$ & $\log \left(T_{\mathrm{b}}\right)$ \\
\hline W3(OH) & 86.6 & 6.8 \\
& 107.0 & 7.8 \\
AFGL 5180 & 107.0 & 7.3 \\
G29.95-0.02 & 85.5 & 6.2 \\
W48 & 107.0 & 7.0 \\
W51-IRS1 & 86.6 & 6.4 \\
G59.78+0.06 & 108.8 & 6.4 \\
DR21(OH) & 85.5 & 6.5 \\
CepA & 107.0 & 7.7 \\
NGC 7538 & 107.0 & 6.9 \\
\hline
\end{tabular}

\section{Methanol rotational temperatures}

96.7 GHz thermal lines are observed in 22 sources out of 23. For each of these 22 sources, the rotational temperature and the beam averaged column density of the methanol cloud are derived by applying the rotation diagram method (Nummelin 1998) to the $96.7 \mathrm{GHz}$ thermal lines only. Methanol populations are usually separated in two species, a singly degenerate A-species and a doubly degenerate E-species. Thus, for excitation purposes the Aand E-species of methanol are independent populations. However, assuming that there is equal abundance of each species is reasonable (Blake et al. 1987). In the rotation diagram analysis, A- and E-species are treated as a single species. The upper level energies $\left(E_{\mathrm{u}}\right)$ are measured from the A-species ground state and are derived from Mekhtiev et al. (1999). The results are given in Table 4. Additionally, the relative abundance of methanol to molecular hydrogen is estimated. The methanol density is calculated by dividing the beam-averaged column density by the size of the source. In all the calculations, a source size of 34 arcsec is assumed. This corresponds to the beam size, i.e. a beamfilling factor of 1 is used to derive the brightness temperature $\left(T_{\mathrm{b}}\right)$ from the antenna temperature $\left(T_{\mathrm{A}}^{*}\right)$. The molecular hydrogen density is set to $10^{6} \mathrm{~cm}^{-3}$ or $10^{7} \mathrm{~cm}^{-3}$ depending on the values of $R_{1,-1}$ which is the line ratio of the $2_{1} \rightarrow 1_{1} \mathrm{E}$ transition to the $2_{-1} \rightarrow 1_{-1} \mathrm{E}$ transition (Menten et al. 1988). $X_{\mathrm{CH}_{3} \mathrm{OH}}$, the relative methanol abundance, is thus the ratio of the methanol density to the molecular hydrogen density.

The methanol rotational temperatures are generally lower than $25 \mathrm{~K}$ with the exception of those in Orion $\mathrm{KL}$ and in W51-IRS1. The temperature values have to be taken with precaution as methanol is believed to be sub-thermally excited (Menten et al. 1988). Thus, the methanol rotational temperature only gives a lower limit of the kinematical temperature in the molecular cloud. The relatively low rotational temperature could mean that the $96.7 \mathrm{GHz}$ transitions trace the outer and cool part of the methanol cloud. Such a result is not surprising. Van Dishoeck \& Blake (1998) have shown, for instance, that methanol forms in hot cores around young massive stars after evaporating from the icy dust mantles at $60 \mathrm{~K}$. In contrast, higher excitation methanol lines would trace the inner, dense and hot part of the molecular core as illustrated by Fig. 2. Indeed, if the methanol clouds are directly surrounding young massive stars, temperature gradients are expected in the molecular core from the hot central core to the outer molecular layer of the protostellar envelope. This has been suggested by Cesaroni et al. (1998) who found clear evidence for temperature gradients in three hot cores using $\mathrm{NH}_{3}$ as a temperature probe. For example, the temperature in G29.95-0.02 decreases from $100 \mathrm{~K}$ at the core central zone to $20 \mathrm{~K}$ at $10000 \mathrm{AU}$ away from the centre.

The derived beam-averaged column densities vary from $10^{14}$ to a few $10^{15} \mathrm{~cm}^{-2}$ with the exception of that in Orion KL. These values are beam-averaged over the telescope beam and are probably underestimated. Highresolution observations of $96.7 \mathrm{GHz}$ thermal emission in the DR21 complex showed that the typical angular size of the methanol clouds varied between $\sim 3^{\prime \prime} \times 3^{\prime \prime}$ and $\sim 10^{\prime \prime} \times 10^{\prime \prime}$ at a distance of $3 \mathrm{kpc}$ (Liechti \& Walmsley 1998). Moreover, if methanol molecules coexist in hot cores with other molecules such as $\mathrm{NH}_{3}$, the typical size of the methanol cloud can be approximated by the diameter 

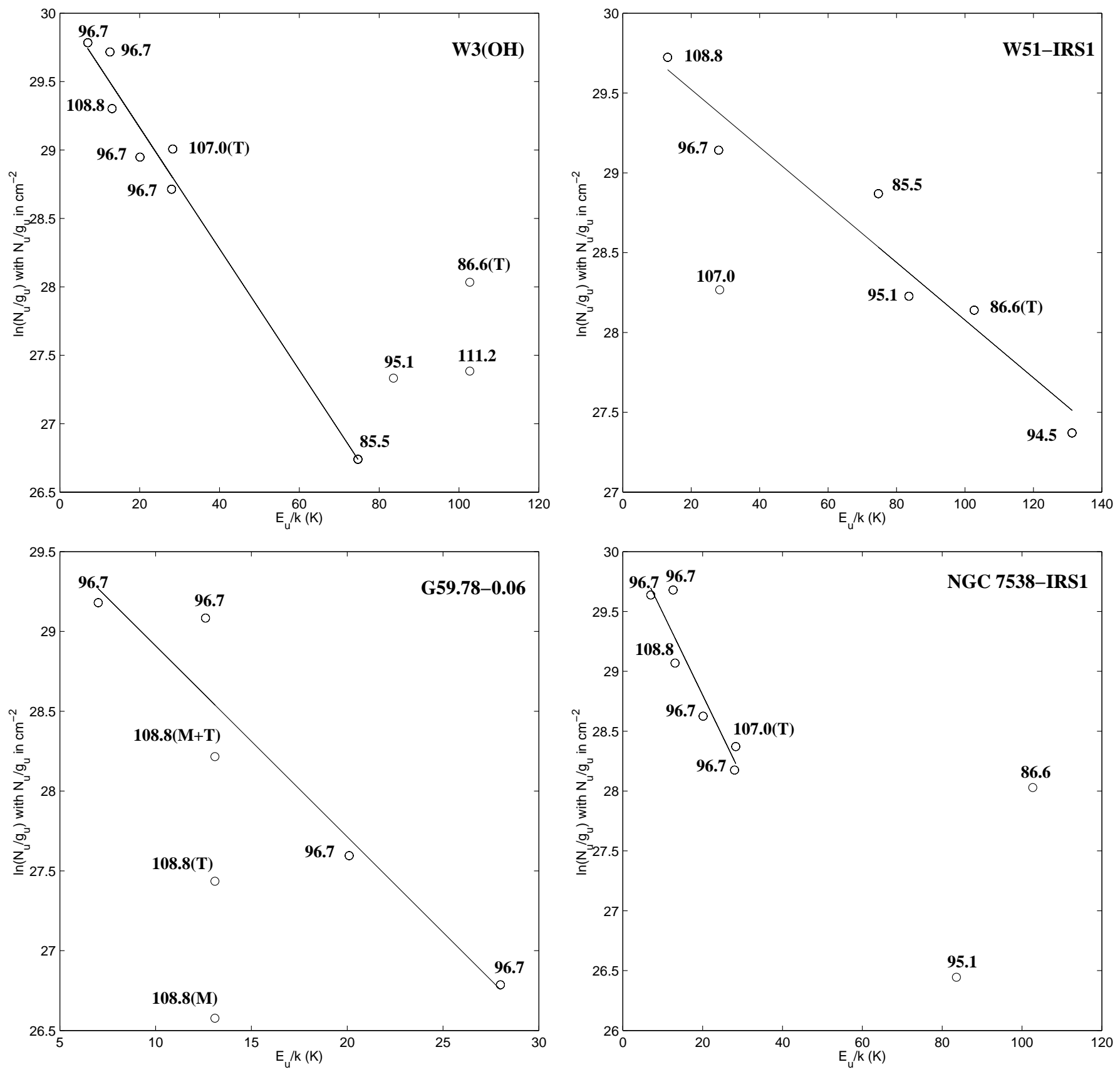

Fig. 1. Rotation diagram for the pedestal components in W3(OH), W51-IRS1, G59.78-0.06 and NGC 7538-IRS1. The angular size used for the source is $34^{\prime \prime}$ corresponding to the telescope beam.

of hot cores which is $\sim 10000$ AU. Hence, the derived column density values have to be multiplied by a corrected factor. Assuming a size of $10000 \mathrm{AU}$ for the methanol cloud, the corrected column densities are listed in Table 4 in the "10000 AU" column. In a similar way, the relative abundance has to be corrected by a large factor (Table 4). After corrections, the relative abundance of methanol is generally in the range $10^{-8}$ to $10^{-6}$.

\section{Class I \& II methanol masers}

95.1 $\mathrm{GHz}$ methanol transition is detected toward 20 sources out of 23 . In 16 sources this transition is masing while thermal emission is found in 13 sources. In 9 sources both $95.1 \mathrm{GHz}$ maser and thermal emission are observed. The $v_{\text {lsr }}$ of thermal emission generally corresponds (9 sources out of 13) to the systemic line-of-sight velocity measured with $\mathrm{NH}_{3}, \mathrm{CS}$ or $\mathrm{C}^{18} \mathrm{O}$ lines (Table A.1). 12 sources exhibit both $6.7 \mathrm{GHz}$ and $95.1 \mathrm{GHz}$ methanol masers (Table A.1). For 7 of them, the $95.1 \mathrm{GHz}$ maser peaks at velocities within the line-of-sight velocity range of the $6.7 \mathrm{GHz}$ masers. In 9 sources $95.1 \mathrm{GHz}$ masers peak at velocities within $1 \mathrm{~km} \mathrm{~s}^{-1}$ around the systemic velocity. Finally, the $6.7 \mathrm{GHz}$ maser velocity is blue shifted or red shifted with respect to systemic velocity in 6 sources: S231, AFGL 5180, S255, IRAS 20126+4104, W75N and CepA. According to the classification of methanol masers, 
Table 4. Rotational temperatures, column densities and relative abundances using $96.7 \mathrm{GHz}$. For $R_{1,-1}<0.5, n_{\mathrm{H}_{2}}=10^{6} \mathrm{~cm}^{-3}$ is assumed to estimate $X_{\mathrm{CH}_{3} \mathrm{OH}}$ and for $R_{1,-1}>0.5, n_{\mathrm{H}_{2}}=10^{7} \mathrm{~cm}^{-3}$. Note: ${ }^{1} T_{\text {rot }}$ and $N_{\mathrm{CH}_{3} \mathrm{OH}}$ in W51-IRS1 have been derived using all the thermal lines.

\begin{tabular}{llllllll}
\hline \hline Sources & $D$ & $T_{\text {rot }}$ & \multicolumn{2}{l}{$N_{\mathrm{CH}_{3} \mathrm{OH}}\left(10^{15} \mathrm{~cm}^{-2}\right)$} & \multicolumn{2}{c}{$X_{\mathrm{CH}_{3} \mathrm{OH}}\left(10^{-9}\right)$} & $R_{1,-1}$ \\
\cline { 5 - 7 } & $(\mathrm{kpc})$ & $(\mathrm{K})$ & $34^{\prime \prime}$ & $10000 \mathrm{AU}$ & $34^{\prime \prime}$ & $10000 \mathrm{AU}$ & \\
\hline W3(OH) & 2.0 & 18 & 1.2 & 57.8 & 1.2 & 386.7 & 0.37 \\
Orion S6 & 0.5 & 21 & 1.7 & 4.8 & 6.5 & 32.1 & 0.39 \\
Orion KL & 0.5 & 137 & 42.5 & 122.9 & 17 & 821.6 & 0.88 \\
OMC 2 & 0.5 & 15 & 0.2 & 0.6 & 0.8 & 3.7 & 0.38 \\
S231 & 2.5 & 9 & 1.0 & 69.6 & 0.8 & 465.7 & 0.13 \\
MonR2 & 0.8 & 18 & 0.1 & 1.1 & 0.4 & 7.4 & 0.29 \\
AFGL 5180 & 2.2 & 8 & 0.4 & 30.0 & 0.4 & 167.0 & 0.08 \\
S255 & 2.5 & 13 & 0.1 & 10.0 & 0.4 & 67.0 & - \\
S269 & 4.0 & - & - & - & - & - & - \\
G29.95-0.02 & 7.5 & 12 & 0.3 & 178.8 & 0.07 & 1195.6 & 0.22 \\
W48 & 3.4 & 23 & 0.1 & 20.3 & 0.09 & 136.0 & 0.44 \\
W51-IRS1 & 7.5 & 55 & 4.8 & 3140.7 & 1.2 & 20999.0 & - \\
W51 e2 & 7.5 & 14 & 5.7 & 3684.9 & 1.5 & 24637.0 & 0.24 \\
W51 met1 & 7.5 & 6 & 0.5 & 313.1 & 0.1 & 2093.3 & 0.003 \\
G59.78+0.06 & 6.9 & 8 & 0.4 & 216.0 & 0.1 & 1444.0 & 0.10 \\
ON1 & 1.4 & 8 & 1.0 & 21.2 & 1.3 & 141.8 & 0.10 \\
IRAS 20126+4104 & 1.7 & 17 & 0.2 & 31.3 & 0.2 & 209.1 & 0.34 \\
ON2 & 5.0 & - & - & - & - & - & - \\
W75N & 2.0 & 10 & 0.6 & 25.8 & 0.5 & 172.4 & 0.20 \\
DR21(OH) & 3.0 & 13 & 1.9 & 200.2 & 1.3 & 1338.7 & 0.22 \\
Cep A & 0.7 & 12 & 0.4 & 2.0 & 1.0 & 13.5 & 0.22 \\
NGC 7538-IRS1 & 2.7 & 13 & 0.9 & 72.2 & 0.6 & 483.0 & 0.23 \\
NGC 7538-44 GHz & 2.7 & 8 & 1.4 & 116.2 & 1.0 & 776.7 & 0.08 \\
\hline & & & & & & & \\
\hline
\end{tabular}

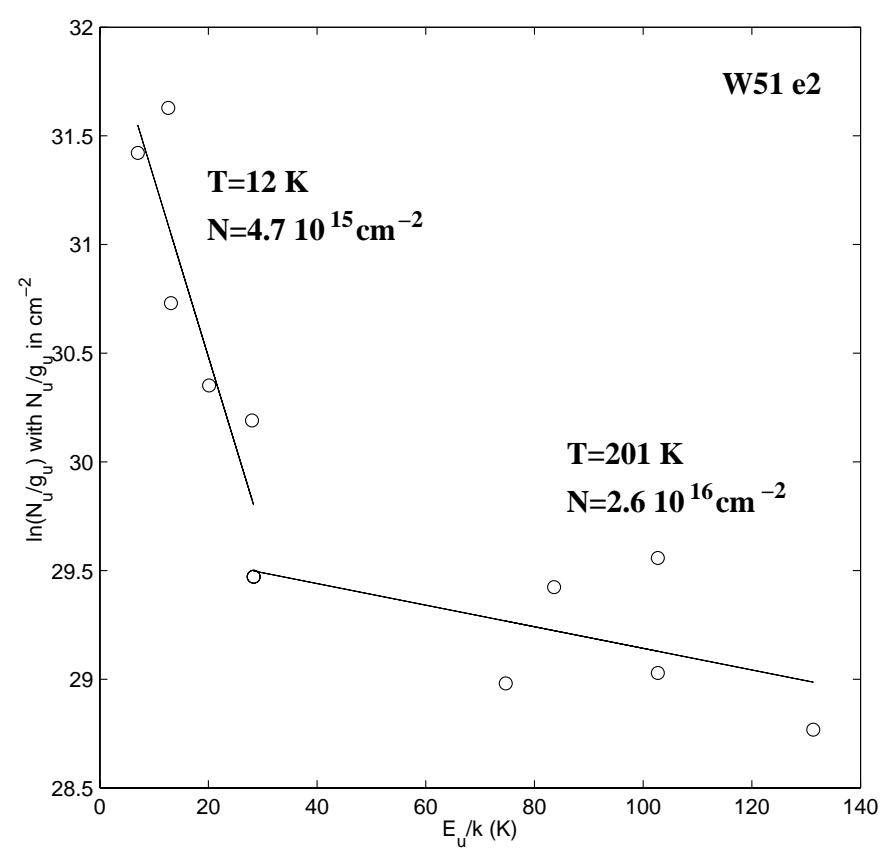

Fig. 2. Rotation diagram for $\mathrm{W} 51 \mathrm{e} 2$. Two rotational temperatures are used to fit the data suggesting the presence of cold and hot methanol gas.

class II $6.7 \mathrm{GHz}$ masers are expected to originate near the young stellar object and thus at velocities around the systemic velocity. Class I 95.1 GHz masers are expected to originate in outflows far away from the young star and thus at velocities blue shifted or red shifted with respect to the systemic velocity. Our results contradict these expectations in many cases.

The fact that 6.7 and $95.1 \mathrm{GHz}$ methanol masers arise at similar velocities in many sources is really puzzling. Could they both originate in the same region? New VLBI evidence at 6.7 and $12.2 \mathrm{GHz}$ suggest that class II methanol masers do form in the inner part of outflows (Minier et al. 2001) which are also the proposed habitat of class I methanol masers. Unfortunately, single dish observations do not give any precise information on the position of the $95.1 \mathrm{GHz}$ emission relatively to the 6.7 GHz maser and coincidence in velocity does not necessarily imply coincidence in position. For instance, the major $95.1 \mathrm{GHz}$ maser spike in NGC 7538-IRS1 is also seen in NGC $7538-44 \mathrm{GHz}$ at the same velocity $\left(-57.51 \mathrm{~km} \mathrm{~s}^{-1}\right)$, but with a stronger intensity and 40 arcsec south to IRS1. Thus strong emission seen with a single dish telescope at two nearby positions (within $\sim$ a telescope beam) and at a same velocity may originate from only one site. It is then impossible to bring any firm conclusion on any spatial relationship between class I and class II masers. Interferometry is needed to solve this puzzle with higher resolution. 
Table 5. $\mathrm{CH}_{3} \mathrm{CN}$ column densities and age of the source deduced from Rodgers \& Charnley (2001). $\mathrm{CH}_{3} \mathrm{CN}$ column densities are derived from the results of Kalenskii et al. (2000) using the rotation method diagram. Notes: ${ }^{1}$ derived from Wilner et al. (1994); ${ }^{2}$ derived from Cesaroni et al. (1997). Mid-IR fluxes are from the MSX catalogue (www.ipac.caltech.edu/ipac/msx/msx.html). $\mathrm{HC}$ and UC H II mean hot molecular core and ultra-compact H II region.

\begin{tabular}{lllllll}
\hline \hline Sources & $N_{\mathrm{CH}_{3} \mathrm{CN}}$ & {$\left[\mathrm{CH}_{3} \mathrm{OH}\right] /$} & Age & \multicolumn{2}{c}{ Mid-IR fluxes $(\mathrm{Jy})$} & Source \\
& $\left(10^{15} \mathrm{~cm}^{-2}\right)$ & {$\left[\mathrm{CH}_{3} \mathrm{CN}\right]$} & $\left(10^{5} \mathrm{yr}\right)$ & $6.8-10.8 \mu \mathrm{m}$ & $18.2-25.2 \mu \mathrm{m}$ & Type \\
\hline W3(OH) & 0.12 & 10 & 1.0 & 69 & 57 & UC H II \\
Orion S6 & 0.07 & 23 & 0.7 & $\mathrm{NO}$ & $\mathrm{NO}$ & $?$ \\
Orion KL & $0.78^{1}$ & 54 & 0.5 & $\mathrm{NO}$ & $\mathrm{NO}$ & $\mathrm{HC}$ \\
S231 & 0.02 & 44 & 0.5 & - & 31 & $?$ \\
W51e2 & 1.56 & 4 & 2.0 & 12 & 294 & HC+UC H II \\
ON1 & 0.01 & 62 & 0.5 & 0.5 & 24 & $?$ \\
IRAS 20126+4104 & $0.007^{2}$ & 25 & 0.7 & 0.8 & 53 & HC \\
W75N & 0.03 & 19 & 0.8 & 15 & 476 & UC H II \\
DR21(OH) & 0.06 & 34 & 0.6 & 9 & 88 & HC \\
CepA & 0.01 & 30 & 0.6 & 5 & 358 & UC H II \\
NGC 7538-IRS1 & 0.02 & 43 & 0.5 & 165 & 1582 & UC H II \\
\hline
\end{tabular}

\section{The ages of the methanol cores}

In Sect. 4, methanol relative abundances of $10^{-8}$ to $10^{-6}$ were derived. These values are in good agreement with recent observational and theoretical results by Rodgers \& Charnley (2001) who have modelled the chemistry at the earliest stages of massive star formation. Assuming initial methanol abundance of $\sim 10^{-6}$, they showed that methanol abundance in a molecular core evolves depending on the initial ammonia abundance and the temperature. Interestingly, their theoretical model suggests that O-bearing and N-bearing molecules can coexist within dense molecular cores and that the abundance ratio of specific O-bearing molecules to N-bearing species could potentially give the age of the core and thus the age of the associated young stellar object. The most interesting Nbearing molecules are $\mathrm{NH}_{3}, \mathrm{HC}_{3} \mathrm{~N}$ and $\mathrm{CH}_{3} \mathrm{CN}$. Kalenskii et al. (2000) observed $\mathrm{CH}_{3} \mathrm{CN}$ in many methanol sources presented in this paper. Using the Gaussian parameters of the $110.3 \mathrm{GHz} \mathrm{CH}_{3} \mathrm{CN}$ lines derived by Kalenskii et al., the column density is estimated with the rotation diagram method (Nummelin 1998). The results are given in Table 5 for the sources where $\mathrm{CH}_{3} \mathrm{CN}$ was detected (see also Table A.1). The column density values are beam-averaged. If methanol and methyl cyanide are present over the same regions in hot cores, the ratio of their densities is simply the ratio of their column densities (Table 5). This ratio is compared to the results from Rodgers \& Charnley with ammonia injected and $T=300 \mathrm{~K}$ (Fig. 4 in Rodgers \& Charnley 2001) and the deduced ages are given in Table 5. The ages range between 0.5 and $2 \times 10^{5}$ years which is consistent with the expected age of young massive stars. This age estimate is uncertain. Nonetheless, it is interesting to note that $\mathrm{W} 3(\mathrm{OH})$ is older than IRAS $20126+4104$ as expected since IRAS $20126+4104$ is in an earlier stage (hot core) than W3(OH) (UC H II). From the mid-infrared fluxes in Table 5, there is no apparent correlation between the mid-infrared luminosity and the estimated ages.
Many assumptions could obviously be sources of errors in our calculations. First, 96.7 GHz methanol and $110.3 \mathrm{GHz}$ methyl cyanide lines are assumed to be optically thin for the purpose of the rotation diagram method. This is uncertain and more refined modelling methods are needed to derive $T_{\text {rot }}$ and $N$. Secondly, the sizes of the $\mathrm{CH}_{3} \mathrm{OH}$ and $\mathrm{CH}_{3} \mathrm{CN}$ cores are assumed to be equal. $\mathrm{CH}_{3} \mathrm{CN}$ could be more confined in the hot core than $\mathrm{CH}_{3} \mathrm{OH}$ and then the density ratio would decrease. Finally, the contamination by other young stellar objects within the large beam of the single dish telescope certainly affects the data. This is likely the case for Orion, W51, W75N, CepA and NGC 7538 where many young massive stars coexist within $\sim 30$ arcsec. Clearly, these results have to be seen as preparatory work to higher resolution observations.

\section{Conclusions}

23 star-forming regions have been searched for new class II methanol maser lines and thermal emission in eleven transitions. This work gives a coherent and global overview of the methanol lines present in massive star-forming regions within the range $85-112 \mathrm{GHz}$. Five masers at $107.0 \mathrm{GHz}$ detected by Val'tts et al. (1995) have been confirmed. New masers at 85.5, 86.6, 95.1 and $108.8 \mathrm{GHz}$ have been detected. However, new class II masers are rare phenomena, and most of the sources exhibit thermal emission or no emission at the frequencies of predicted masers.

Finally, assuming that methanol emission originates in molecular cores surrounding young massive stars, their ages is estimated to $\sim 10^{5}$ years. Future interferometric observations are needed to better constrain the size, the maser brightness temperature, rotational temperature, column densities and ages of the methanol clouds.

Acknowledgements. The authors thank the Onsala-20 m telescope staff as well as Jiyune Yi and Michele Pestalozzi for their help during the observations. We also thank Dinah Cragg for her valuable advice on methanol line analysis. 


\section{Appendix A: Gaussian parameters and spectra of the methanol lines}

The Gaussian parameters of the detected methanol lines are listed in Table A.1. Complementary information from the literature and from this work about other species detected toward the selected sources is also listed. Methanol line spectra at the observed frequencies are presented in Figs. A.1 and A.2. The velocity scale is identical in all spectra.

\section{References}

Anglada, G., Estalella, R., Pastor, J., Rodriguez, L. F., \& Haschick, A. D. 1996, ApJ, 463, 205

Bachiller, R., Gomez-Gonzalez, J., Barcia, A., \& Menten, K. M. 1990, A\&A, 240, 116

Batrla, W., Wilson, T. L., Ruf, K., \& Bastien, P. 1983, A\&A, 128,279

Batrla, W., Matthews, H. E., Menten, K. W., \& Walmsley, C. M. 1987, Nature, 326, 49

Blake, G. A., Sutton, E. C., Masson, C. R., \& Phillips, T. G. 1987, ApJ, 315, 621

Caswell, J. L., Vaile, R. A., Ellingsen, S. P., Whiteoak, J. B., \& Norris, R. P. 1995, MNRAS, 272, 96

Caswell, J. L., Yi, Jiyune, Booth, R. S., \& Cragg, D. M. 2000, MNRAS, 313, 599

Cesaroni, R., Felli, M., Testi, L., Walmsley, C. M., \& Olmi, L. 1997, A\&A, 325, 725

Cesaroni, R., Hofner, P., Walmsley, C. M., \& Churchwell, E. 1998, A\&A, 331, 709

Chini, R., Reipurth, B., Ward-Thompson, D., Bally, J., et al. 1997, ApJ, 474, L135

Cragg, D. M., Sobolev, A. M., Ellingsen, S. P., et al. 2000, MNRAS, 323, 939

Haschick, A. D., \& Baan, W. A. 1989, ApJ, 339, 949

Haschick, A. D., Menten, K. M., \& Baan, W. A. 1990, ApJ, 354,556

Hofner, P., Wyrowski, F., Walmsley, C. M., \& Churchwell, E. 2000, ApJ, 536, 393

Hofner, P., et al. 2002, in preparation

Kalenskii, S. V., Promislov, V. G., Alakoz, A., Winnberg, A. V., \& Johansson, L. E. B. 2000, A\&A, 354, 1036
Kalenskii, S. V., Slysh, V. I., Val'tts, I. E., Winnberg, A., \& Johansson, L. E. 2001, Astron. Rep., 45, 26

Liechti, S., \& Walmsley, C. M. 1998, A\&A, 321, 625

Mangum, J. G., Wootten, A., \& Mundy, L. G. 1991, ApJ, 378, 576

Mangum, J. G., Wootten, A., \& Mundy, L. G. 1992, ApJ, 388, 467

Mekhtiev, M. A., Godfrey, P. D., \& Hougen, J. T. 1999, J. Mol. Spec., 194, 171

Menten, K. M., \& Batrla, W. 1989, ApJ, 341, 839

Menten, K. M. 1991a, in ASP Conf. Ser. 16, Atoms, ions and molecules: New results in spectral line astrophysics, ed. A. D. Haschick, \& P. T. P. Ho, 119

Menten, K. M. 1991b, ApJ, 380, L75

Menten, K. M., Walmsley, C. M., Henkel, C., \& Wilson, T. L. 1988, A\&A, 198, 253

Minier, V., Booth, R. S., \& Conway, J. E. 2000, A\&A, 362, 1093

Minier, V., Conway, J. E., \& Booth, R. S. 2001, A\&A, 369, 278

Minier, V., Booth, R. S., \& Conway, J. E. 2002, A\&A, 383, 614 (MBC02)

Nummelin, A. 1998, Ph.D. Thesis, Technical Report No. 347, Chalmers University of Technology

Plambeck, R. L., \& Wright, M. C. H. 1988, ApJ, 330, L61

Rodgers, S. D., \& Charnley, S. B. 2001, ApJ, 546, 324

Sobolev, A. M. 1993, PAZh, 19, 72

Sobolev, A. M., Cragg, D. M., \& Godfrey, P. D. 1997, MNRAS, 288, L39

Sutton, E. C., Sobolev, A. M., Ellingsen, S. P., et al. 2001, ApJ, 554, 173

Turner, B. E., Gordon, M. A., \& Wrixon, G. T. 1972, ApJ, 177,609

Val'tts, I. E., Dzura, A. M., Kalenskii, S. V., et al. 1995, A\&A, 294,825

Val'tts, I. E., Ellingsen, S. P., Slysh, V. I., et al. 1999, MNRAS, 310,1077

Val'tts, I. E., Ellingsen, S. P., Slysh, V. I., et al. 2000, MNRAS, 317,315

van Dishoeck, E. F., \& Blake, G. A. 1998, ARA\&A, 36, 317

Wilner, D. J., Wright, M. C. H., \& Plambeck, R. L. 1994, ApJ, 422,642

Wilson, T. L., Serabyn, E., Henkel, C., \& Walmsley, C. M. 1986, A\&A, 158, L1

Zhang, Q., Hunter, T. R., \& Sridharan, T. K. 1998, ApJ, 505, L151 